\title{
Morphologic Study of the Lateral and Medial Collateral Ligaments of the Human Knee
}

\author{
By \\ Norihiro OTAKE, Huayue CHEN, Xianfeng YAO and Shizuko SHOUMURA \\ Department of Anatomy, Gifu University Graduate School of Medicine, 1-1 Yanagido, Gifu, 501-1194, Japan
}

- Received for Publication, November 8, $2006-$

Key Words: Knee, Lateral collateral ligament, Medial collateral ligament, Morphology, Human gross anatomy

\begin{abstract}
Summary: The collateral ligaments of the knee are important in maintaining knee stability. Numerous studies have been described the morphology and function of the ligaments of the knee. However, little data was given about the lateral and medial collateral ligaments simultaneously. The objective of the present study was to compare the morphology of the lateral (LCL) and medial (MCL) collateral ligaments in human knees. We dissected 34 cadaver knees and examined the size, attachment and inclination of the LCL and the MCL. In both of them, the posterior bundle was longer than that of the anterior bundle. The anterior and posterior bundles of the MCL were longer than that of the LCL. The proximal attachment of the MCL situated more anterior and superior than that of the LCL. As compared with the LCL, the distal attachment of the MCL located a little forward and inferior. The longitudinal diameter of the attachment of ligaments was longer than the transverse one, except for the proximal attachment of the MCL. The size of the attachments of the MCL was larger than that of the LCL except for the longitudinal diameter of the proximal attachment. At full extension of the knee, the inclination of the MCL was backward, while that of the LCL was forward. These data showed that the LCL and MCL had the different structural properties. It may provide a better understanding of the function of the collateral ligaments.
\end{abstract}

The human knee collateral ligaments play an important role in maintaining knee stability (Kapandji, 1983; Grood et al., 1988; Liow et al., 2003; Robinson et al., 2004; Park et al., 2005; EspregueiraMendes and da Silva, 2006; Wymenga et al., 2006). The medial collateral ligament (MCL) may be injured alone or in combination with the anterior cruciate ligament (ACL). Injury of the lateral collateral ligament (LCL) often accompanies ruptures of the posterior cruciate ligament (PCL). There have been a number of studies concerning the morphology and biomechanics of the LCL and MCL (Warren et al., 1974; Bartel et al., 1977; Nomura et al., 1989; Sugita and Amis, 2001; Robinson et al., 2004; Espregureira-Mendes and da Silva, 2006). Although the function of the LCL is somewhat complex, the primary role is to restrain abnormal motion. Many studies have shown that the $\mathrm{LCL}$ is the major restraint to primary varus rotation at all positions of knee flexion (Warren and Marshall, 1979; Grood et al., 1988; Suda et al., 1993; Shahne et al., 1999; Meister et al., 2000; Sugita and Amis, 2001; Espregueira-Mendes and da Silva, 2006). The MCL complex has recently been divided into three main structures: the longitudinal fibers of the superficial MCL, the deep MCL (Fetto and Marshall, 1978) and the posteromedial capsule (Robinson et al., 2004). Previous studies have treated the MCL as a whole and did not distinguish the different parts of the ligament. Some anatomical studies, however, identified them (Kennedy et al., 1976; Murase et al., 1985; Robinson et al., 2005). They reported the structural properties of these three individual structures (Robinson et al., 2005). In the present study, we examined the size, attachment and inclination of the superficial MCL, not deep one. The MCL has been shown to resist valgus moments and anterior loads beyond $60^{\circ}$ of flexion (Robinson et al., 2004). The MCL has also been considered to stabilize the knee in response to anterior tibial loads after ACL injury. It was described that the length of the MCL and LCL changed with knee flexion (Bartel et al., 1977; Park et al., 2005). There are few reports concerning the

Correspondence to: Norihiro Otake, Department of Anatomy, Gifu University Graduate School of Medicine, 1-1 Yanagido, Gifu, 501-1194, Japan. 


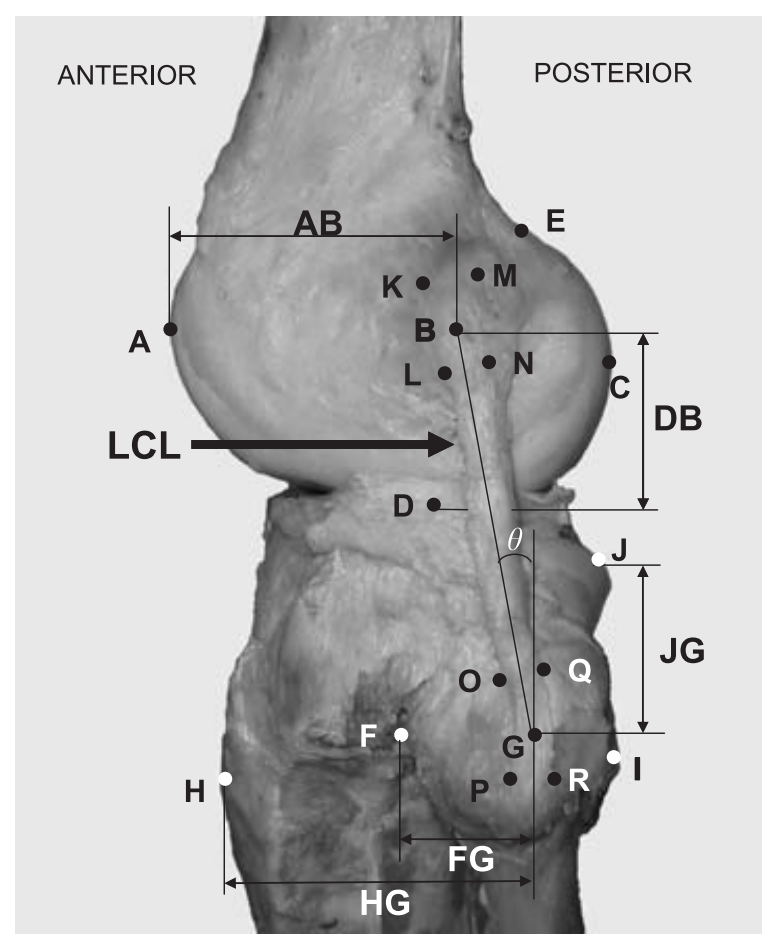

$\alpha$

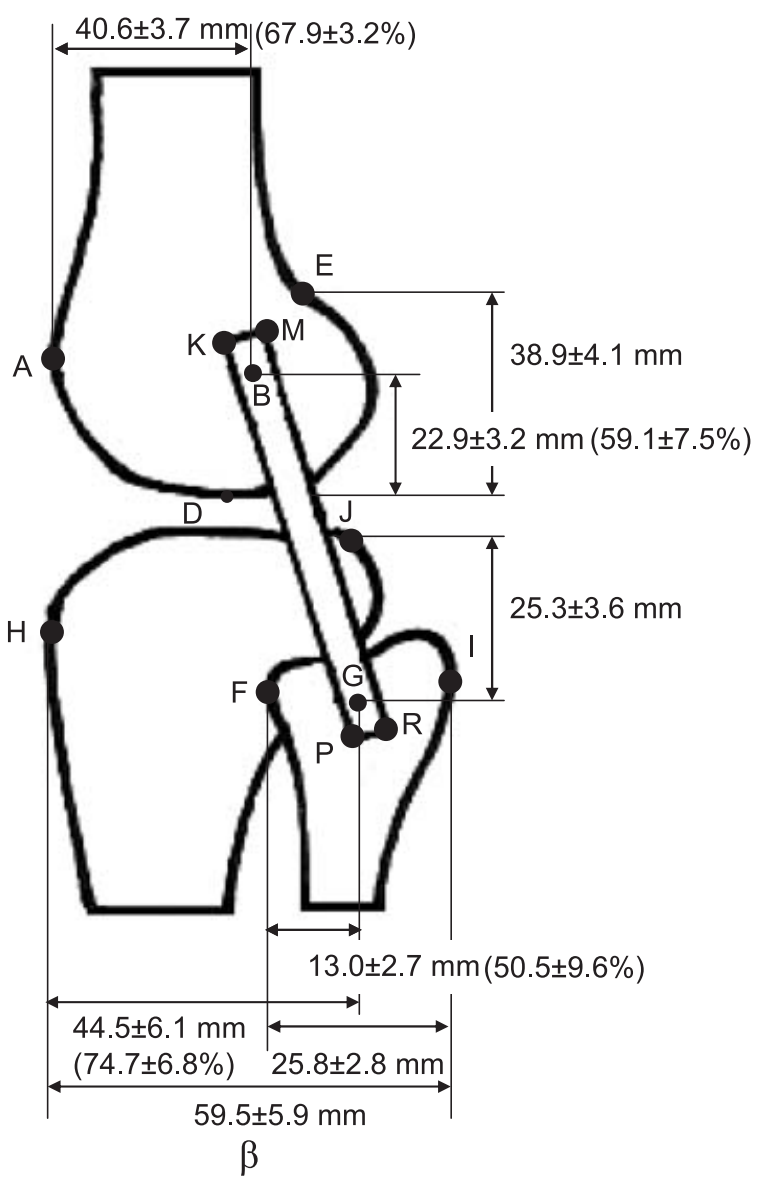

Fig. 1. Attachment site of the LCL of the left knee joint. Fig. 1 $\alpha$ : Photograph. Fig. 1 $\beta$ : Schematic drawing. A: the anterior margin of the lateral condyle; B: the center of the proximal attachment; $\mathrm{C}$ : the posterior margin of the lateral condyle; D: the inferior margin of the lateral condyle; E: the anterior margin of the lateral condyle; F: the anterior margin of the fibular head; G: the center of the distal attachment; H: the anterior margin of the tibia; I: the posterior margin of the fibular head; J: the superior margin of the lateral tibial plateau; $\theta$ : the inclination of the longitudinal axis of the LCL.

LCL and MCL simultaneously the same knee. The purpose of the present study was to investigate and compare the structural properties of the LCL and MCL of the same knee.

\section{Materials and Methods}

We used 18 right and 16 left knees from 6 male and 17 female cadavers. They showed no particular pathologic findings on the bone and joint. The average age of cadavers was $79.5 \pm 9.5$ years (age range 64-98 years old). The bones were cut about $150 \mathrm{~mm}$ above and below the knee joint. The muscles were removed from the femur, tibia and fibula, preserving the ligaments and joint capsule intact. When the LCL and MCL were identified, the pattern and definition of the structures were examined. At full extension of the knee joint, we measured and recorded the position and the size of the at- tachments and the length of the LCL (Fig. 1 $\alpha$ ) and MCL (Fig. $2 \alpha$ ) by using a slide caliper, as followed. In the sagittal plane, we measured (i); the anteroposterior distance of the lateral femoral condyle (Fig. 1 $\alpha$, AC), and (ii); the distance of the center of proximal attachment of the LCL from the anterior margin of the lateral femoral condyle (Fig. 1 $\alpha, A B$ ). The center of the proximal attachment was shown as the percentage (ii/i). We measured (iii); the superoinferior distance of the lateral femoral condyle from the inferior margin (Fig. 1 $\alpha, \mathrm{DE}$ ) and (iv); the distance of the center of proximal attachment of the LCL from the inferior margin of the lateral femoral condyle (Fig. 1 $\alpha, \mathrm{DB}$ ). The center of the proximal attachment was shown as the percentage (iv/iii). We measured (v); the anteroposterior distance of the fibular head (Fig. 1 $\alpha$, FI) and (vi); the distance of the center of distal attachment of the LCL from the anterior margin of the fibular head (Fig. 1 $\alpha$, FG). Furthermore, we measured (vii); the ante- 


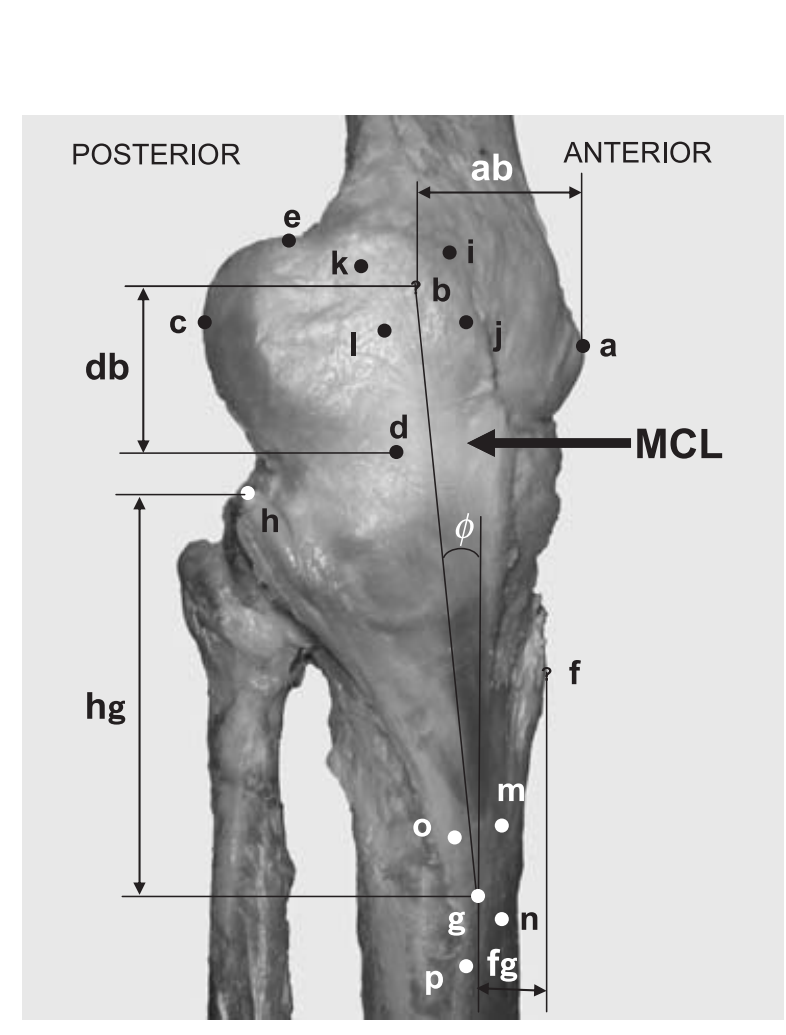

$\alpha$

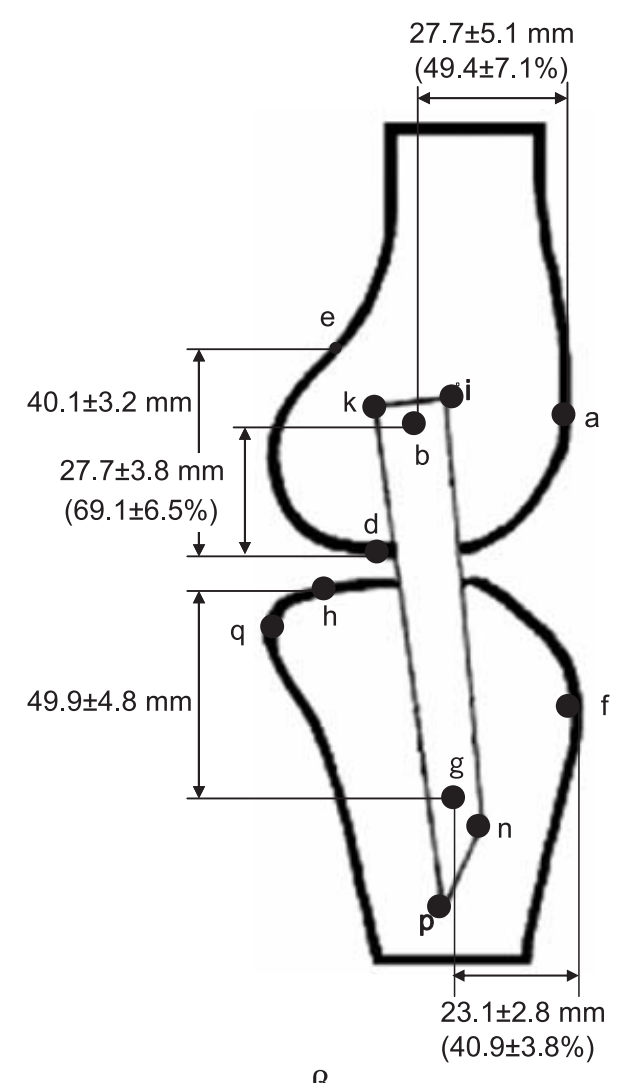

$\beta$

Fig. 2. Attachment site of the MCL of the left knee joint. Fig. 2 $\alpha$ : Photograph. Fig. 2 $\beta$ : Schematic drawing. a: the anterior margin of the medial condyle; $b$ : the center of the proximal attachment; $c$ : the posterior margin of the medial condyle; d: the inferior margin of the medial condyle; e: the anterior margin of the medial condyle; f: the anterior margin of the tibia; g: the center of the distal attachment; $h$ : the superior margin of the medial tibial plateau; $\phi$ : the inclination of the longitudinal axis of the MCL.

roposterior distance between anterior margin of the tibia and posterior margin of the fibular head (Fig. $1 \alpha, \mathrm{HI})$ and (viii); the distance of the center of distal attachment of the LCL from the anterior margin of the tibia (Fig. 1 $\alpha, \mathrm{HG}$ ). The center of the distal attachment was shown as the percentage (vi/v), (viii/vii). We measured the superoinferior distance of the center of distal attachment of the LCL from the lateral plateau of tibia (Fig. $1 \alpha, \mathrm{JG}$ ). We measured and calculated them about the MCL by the same way (Fig. 2 $\alpha$ ). We measured the length of the anterior and posterior bundles of the LCL and MCL (Figs. 3, 4) and the size of the attachment area of the LCL and MCL (Figs. 5, 6). We measured the longitudinal and transverse diameters of the proximal attachment of the LCL and MCL respectively. The angle between the longitudinal axis of the collateral ligaments and the long axis of the tibia (inclination of the longitudinal axis of the LCL, MCL) was measured. Furthermore, the angle between the attachment bundles and the frontal plane or the long axis of the tibia (inclination of the proximal and distal attachment bundle of the LCL, MCL) was evaluated with a protractor.

All data were presented as means \pm SD. The statistical analysis was performed using unpaired t-test (Microsoft Office Excel). $\mathrm{P}$ values of $<0.05$ in the analyses were considered as statistically significant.

\section{Results}

1) The shape of the proximal and distal attachments of the LCL and MCL

The LCL was a cord-like ligament, extending inferiorly from the lateral epicondyle of the femur to the lateral surface of the fibular head. It had a semicircular-shaped attachment on the femoral condyle, with well-defined limits. The fibular attachment of the LCL had a fan-like shape. Its most lateral fibers continued distally as a reinforcement of the fascia over the peroneus longus muscle of the lateral compartment of the leg (Fig. 1 $\alpha$ ). The MCL 


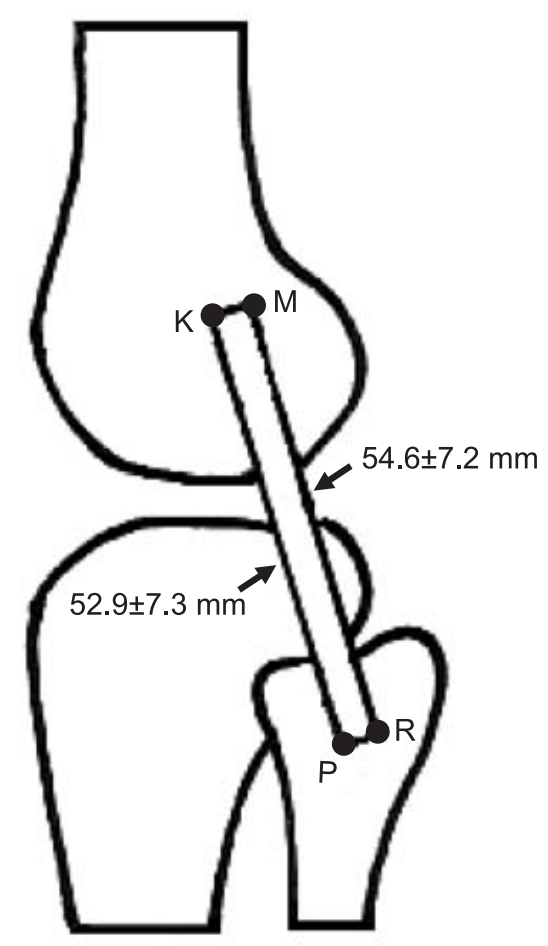

Fig. 3. The length of the LCL of the left knee joint. KP: the anterior length of the bundle; MR: the posterior length of the bundle.

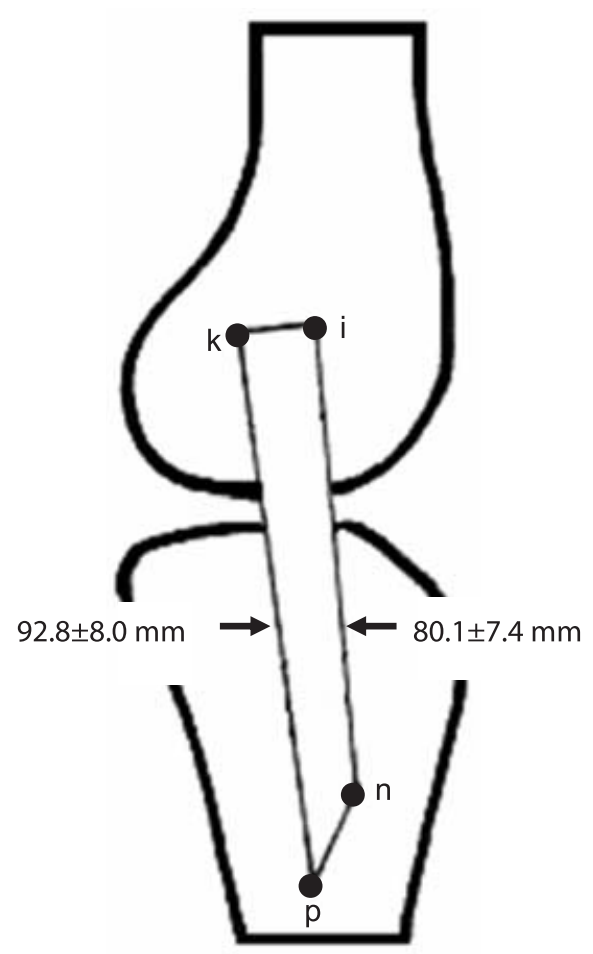

Fig. 4. The length of the MCL of the left knee joint. in: the anterior length of the bundle; $\mathrm{kp}$ : the posterior length of the bundle.

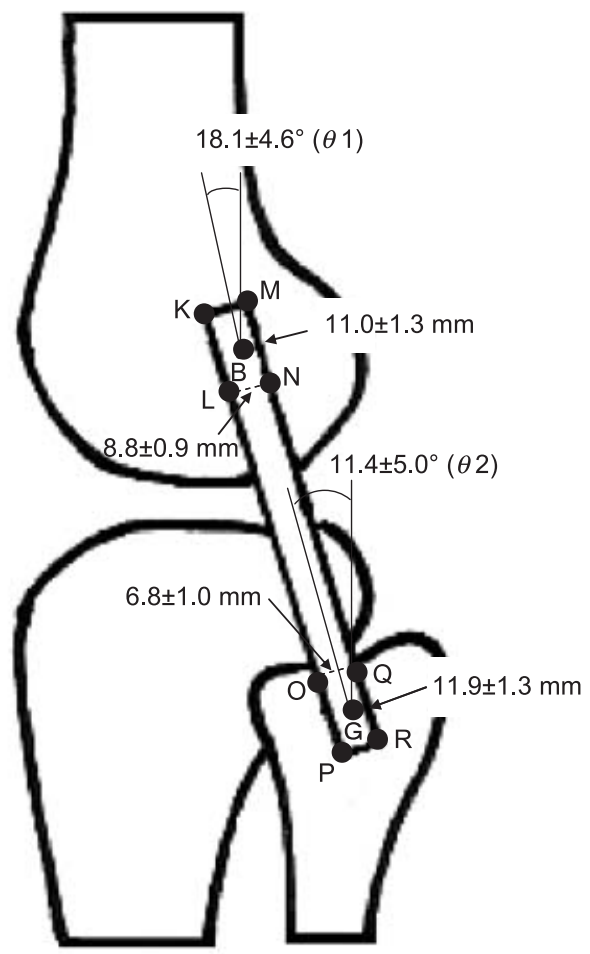

Fig. 5. The size of the LCL attachment area of the left knee joint. MN: longitudinal diameter of the femoral attachment; LN: transverse diameter of the proximal attachment; QR: longitudinal diameter of the distal attachment; OQ: transverse diameter of the distal attachment; KLNM: proximal attachment area; B: the center of the proximal attachment; OPRQ: distal attachment area; G: the center of the distal attachment; $\theta 1$ : the inclination of the proximal attachment bundle of the LCL; $\theta 2$ : the inclination of the distal attachment bundle of the LCL.

was a flat band that extended from the medial epicondyle of the femur to the medial condyle and the superior part of the medial surface of the tibia. The proximal attachment of the MCL was oval in shape and the distal attachment had a trapezoid shape (Fig. $2 \alpha$ ).

2) The proximal and distal attachments of the LCL (Fig. 1)

The mean distance between the anterior and posterior margins of the lateral femoral condyle was $59.9 \pm 5.0 \mathrm{~mm}$ (range $50.2-69.0 \mathrm{~mm}$, Fig. $1 \alpha$, AC). The mean distance between the anterior margin of the lateral femoral condyle and the center of the proximal attachment of the LCL was $40.6 \pm 3.7 \mathrm{~mm}$ (range $33.7-48.0 \mathrm{~mm}$, Fig. $1 \beta, A B$ ). The center of the femoral attachment was $67.9 \pm 3.2 \%$ posterior from the anterior margin of the lateral condyle (Fig. 1, AB). 


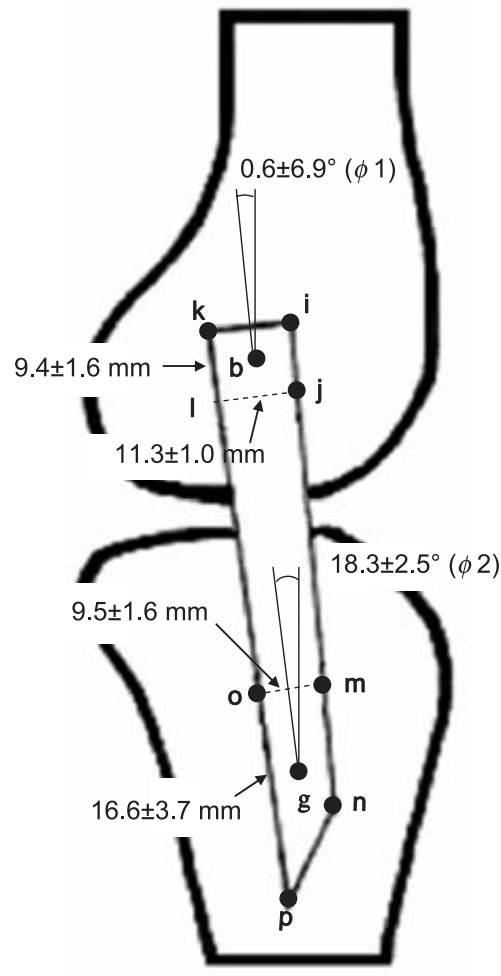

Fig. 6. The size of the MCL attachment area of the left knee joint. kl: longitudinal diameter of the proximal attachment; jl: transverse diameter of the proximal attachment; op: longitudinal diameter of the distal attachment; mo: transverse diameter of the distal attachment; ijlk: proximal attachment area; b: the center of the proximal attachment; mnpo: distal attachment area; g: the center of the distal attachment; $\phi 1$ : the inclination of the proximal attachment bundle of the MCL; $\phi 2$ : the inclination of the distal attachment bundle of the MCL.

The mean distance between the superior and inferior margin of the lateral femoral condyle was $38.9 \pm 4.1 \mathrm{~mm}$ (range 33.0-47.0 mm, Fig. 1 $\beta, \mathrm{DE}$ ). The mean distance between the inferior margin of the lateral femoral condyle and the center of the proximal attachment was $22.9 \pm 3.2 \mathrm{~mm}$ (range $15.0-31.0 \mathrm{~mm}$, Fig. $1 \beta, \mathrm{DB})$. The center of the proximal attachment was $59.1 \pm 7.5 \%$ superior from the inferior margin of the lateral femoral condyle.

The mean distance between the anterior and posterior margin of the fibular head was $25.8 \pm 2.8 \mathrm{~mm}$ (range $23.0-32.0 \mathrm{~mm}$, Fig. $1 \beta, \mathrm{FI}$ ). The mean distance between the anterior margin of the fibular head and the center of the distal attachment was $13.0 \pm 2.7 \mathrm{~mm}$ (range $8.0-20.0 \mathrm{~mm}$, Fig. $1 \beta, F G)$. The center of the distal attachment was $50.5 \pm 9.6 \%$ posterior from the anterior margin of the fibular head.

The mean distance between the anterior margin of the tibia and the posterior margin of the fibular head was $59.5 \pm 5.9 \mathrm{~mm}$ (range $48.0-70.0 \mathrm{~mm}$, Fig. $1 \beta, \mathrm{HI})$. The mean distance between the anterior margin of the tibia and the center of the distal attachment was $44.5 \pm 6.1 \mathrm{~mm}$ (range $31.0-59.0 \mathrm{~mm}$, Fig. $1 \beta, H G)$. The center of the distal attachment was $74.7 \pm 6.8 \%$ posterior from the anterior margin of the tibia. The mean distance between the superior margin of the lateral tibial plateau and the center of the distal attachment was $25.3 \pm 3.6 \mathrm{~mm}$ (range 19.0-35.0 mm, Fig. 1 $\beta, \mathrm{JG}$ ).

3) The proximal and distal attachments of the MCL (Fig. 2)

The mean distance between the anterior and posterior margin of the medial femoral condyle was $56.1 \pm 6.5 \mathrm{~mm}$ (range 33.2-67.0 mm, Fig. $2 \alpha$, ac). The mean distance between the anterior margin of the medial femoral condyle and the center of the proximal attachment of the MCL was $27.7 \pm 5.1 \mathrm{~mm}$ (range 18.4-39.7 mm, Fig. $2 \beta$, ab). The center of the proximal attachment was $49.4 \pm 7.1 \%$ posterior from the anterior margin of the medial femoral condyle.

The mean distance between the superior and inferior margin of the medial femoral condyle was $40.1 \pm 3.2 \mathrm{~mm}$ (range $35.0-47.0 \mathrm{~mm}$, Fig. $2 \beta$, de) The mean distance between the inferior margin of the medial femoral condyle and the center of the proximal attachment was $27.7 \pm 3.8 \mathrm{~mm}$ (range $21.0-38.0 \mathrm{~mm}$, Fig. $2 \beta, \mathrm{db}$ ). The center of the proximal attachment was $69.1 \pm 6.5 \%$ superior from the inferior margin of the medial femoral condyle.

The mean distance between the anterior and posterior margin of the tibia was $56.6 \pm 4.8 \mathrm{~mm}$ (range $48.0-65.0 \mathrm{~mm}$, Fig. $2 \beta$, fq). The mean distance between the anterior margin of the tibia and the center of the distal attachment was $23.1 \pm 2.8 \mathrm{~mm}$ (range 18.0-29.0 mm, Fig. $2 \beta$, fg). The center of the distal attachment was $40.9 \pm$ $3.8 \%$ posterior from the anterior margin of the tibia. The mean distance between the superior margin of the medial tibial plateau and the center of the distal attachment was $49.9 \pm 4.8 \mathrm{~mm}$ (range 41.0 $64.0 \mathrm{~mm}$, Fig. $2 \beta, \mathrm{hg}$ ).

4) Comparison of the attachments of the LCL and MCL

The proximal attachment of the LCL was $67.9 \%$ posterior, while that of the MCL was $49.4 \%$ posterior from the anterior margin of the femoral condyle. The center of the proximal attachment of the MCL was more anterior than that of the LCL $(p<0.05)$. The proximal attachment of the LCL was $59.1 \%$ superior, while that of the MCL was $69.1 \%$ superior from the inferior margin of the 
femoral condyle. The proximal attachment of the MCL was more superior than that of the LCL $(\mathrm{p}<0.05)$.

The distal attachment of the LCL was $50.5 \%$ posterior from the anterior margin of the fibular head and $74.7 \%$ posterior from the anterior margin of the tibia. The distal attachment of the MCL was $40.9 \%$ posterior from the anterior margin of the tibia. The distal attachment of the LCL situated just at the center of the fibular head, while that of the MCL was a little forward.

The distal attachment of the LCL was $25.3 \mathrm{~mm}$ inferior, while that of the MCL was $49.9 \mathrm{~mm}$ inferior from the superior margin of the tibial plateau. The distal attachment of the MCL was more inferior than that of the LCL $(\mathrm{p}<0.05)$.

5) The length of the anterior and posterior bundles of the LCL and MCL (Figs. 3, 4)

The mean length of the anterior and posterior bundles of the LCL was $52.9 \pm 7.3 \mathrm{~mm}$ (range $35.6-70.0 \mathrm{~mm}$ ) and $54.6 \pm 7.2 \mathrm{~mm}$ (range $37.8-$ $73.0 \mathrm{~mm}$ ) respectively (Fig. 3). The mean length of the anterior and posterior bundles of the MCL was $80.1 \pm 7.4 \mathrm{~mm}$ (range $70.0-100.0 \mathrm{~mm}$ ) and $92.2 \pm 8.0 \mathrm{~mm}$ (range $76.8-110.0 \mathrm{~mm}$ ) respectively (Fig. 4). The length of the MCL was significantly longer than that of the LCL for both anterior and posterior bundles $(p<0.05)$. The length of the posterior bundle of the MCL was significantly longer than that of the anterior one $(p<0.05)$. There was no significant difference between the length of the anterior and posterior bundles of the LCL.

6) The size of the attachment area of the LCL and MCL (Figs. 5, 6)

The longitudinal and transverse diameters of the proximal attachment of the LCL were $11.0 \pm 1.3 \mathrm{~mm}$ (range $8.0-13.0 \mathrm{~mm}$ ) and $8.8 \pm$ $0.9 \mathrm{~mm}$ (range $7.0-10.0 \mathrm{~mm}$ ) respectively (Fig. 5, $\mathrm{MN}, \mathrm{LN}$ ). The longitudinal diameter of the LCL attachment was significantly longer than that of the transverse one $(\mathrm{p}<0.05)$.

The longitudinal and transverse diameters of the proximal attachment of the MCL were $9.4 \pm$ $1.6 \mathrm{~mm}$ (range $6.8-13.0 \mathrm{~mm}$ ) and $11.3-1.0 \mathrm{~mm}$ (range 9.7-14.0 mm) respectively (Fig. 6, kl, jl). The transverse diameter of the MCL attachment was significantly longer than that of the longitudinal one $(\mathrm{p}<0.05)$.

The longitudinal and transverse diameters of the distal attachment of the LCL were $11.9 \pm 1.3 \mathrm{~mm}$ (range $10.0-16.0 \mathrm{~mm}$ ) and $6.8 \pm 1.0 \mathrm{~mm}$ (range $5.0-10.0 \mathrm{~mm}$ ) respectively (Fig. 5, QR, OQ). The longitudinal diameter of the LCL attachment was significantly longer than that of the transverse one $(\mathrm{p}<0.05)$.

The longitudinal and transverse diameters of the distal attachment of the MCL were $16.6 \pm 3.7 \mathrm{~mm}$ (range $10.0-25.0 \mathrm{~mm}$ ) and $9.5 \pm 1.6 \mathrm{~mm}$ (range $7.0-15.0 \mathrm{~mm}$ ) respectively (Fig. 6, op, mo). The longitudinal diameter of the MCL attachment was significantly longer than that of the transverse one $(\mathrm{p}<0.05)$.

The size of the MCL attachment was larger than that of the LCL attachment, except for the longitudinal diameter of the femoral attachment.

7) The inclination of the LCL and MCL (Figs. 5, 6) The angle between the proximal attachment of the LCL and the frontal plane $(\theta 1)$ was $18.1 \pm 4.6^{\circ}$ (range $5-30^{\circ}$ ) and the angle between the distal attachment of the LCL and the tibial longitudinal axis $(\theta 2)$ was $11.4 \pm 5.0^{\circ}$ (range $3-25^{\circ}$ ) in the sagittal plane (Fig. 5). The proximal and distal attachment bundles of the LCL showed the forward inclination. The angle between the longitudinal axis of the LCL and the tibial longitudinal axis was $16.7 \pm 4.6^{\circ}$ (range $10-27^{\circ}$, Fig. $1 \alpha, \theta$ ), showing the forward inclination.

The angle between the proximal attachment of the MCL and the frontal plane $(\phi 1)$ was $0.6 \pm 6.9^{\circ}$ (range forward $10^{\circ}$-backward $10^{\circ}$ ). The angle between the distal attachment of the MCL and the tibial longitudinal axis $(\phi 2)$ was $18.3 \pm 2.5^{\circ}$ (range 15-23․ Fig. 6), demonstrating the backward inclination. The angle between the longitudinal axis of the MCL and the tibial longitudinal axis was $19.1 \pm 3.6^{\circ}$ (range $10-23^{\circ}$, Fig. $2 \alpha, \phi$ ) and revealed the backward inclination.

\section{Discussion}

The anatomy of the lateral and medial aspects of the human knee is complex arrangement of static (ligaments) and dynamic (tendons and muscles) stabilizing structures (Warren et al., 1979; Sugita et al., 2001; Pasque et al., 2003; Robinson et al., 2004; Espregueria-Mendes and da Silva, 2006). Few studies have compared the structural properties of the LCL and MCL (Park et al., 2005). The present study examined and compared the morphology of the LCL and MCL in the same knee.

The LCL was a rounded bundle and extended from the lateral epicondyle of the femur to the lateral surface of the fibular head. It had a semicircular-shaped attachment on the femoral condyle. The distal attachment of the LCL had a fan-like shape (Espregueria-Mendes and da Silva, 2006). The superficial MCL was a flat sheet and 
extended from the medial epicondyle of the femur to the medial condyle and the superior part of the medial surface of the tibia. The proximal attachment of the MCL was oval in shape and the distal attachment had a trapezoid shape (Robinson et al., 2004). These were similar to our result.

In the present study, the mean distance between the anterior and posterior margin of the lateral femoral condyle was about $60 \mathrm{~mm}$ and that of the medial femoral condyles was about $56 \mathrm{~mm}$. We found that the proximal attachment of the LCL was $68 \%$ posterior from the anterior margin of the lateral femoral condyle. Similar results were found in the literatures (Suda et al., 1992; EspregueriaMendes and da Silva, 2006). The proximal attachment of the MCL was about $50 \%$ posterior from the anterior margin of the medial femoral condyle (Nomura et al., 1989). The distal attachment of the LCL was about $50 \%$ posterior from the anterior margin of the fibular head, situated just at the center of the fibular head (Suda et al., 1992). It was described that the proximal tibiofibular joint was variable and this could be correlated with the functions of the soft tissue structures attaching to the fibular head (Sugita and Amis, 2001).

In the present study, we found that the mean distance between the superior and inferior margin of the lateral and medial femoral condyles was almost the same, about $40 \mathrm{~mm}$. The proximal attachment of the LCL was about $60 \%$ superior from the inferior margin of the lateral femoral condyle. The proximal attachment of the MCL was more superior than that of the LCL, at about $70 \%$ superior from the inferior margin of the medial femoral condyle. The mean distance between the superior margin of the lateral tibial plateau and the distal attachment of the LCL was $25 \mathrm{~mm}$. The mean distance between the superior margin of the medial tibial plateau and the distal attachment of the MCL was $50 \mathrm{~mm}$. The distal attachment of the MCL located more inferior than that of the LCL. We expect that these data are useful for localizing the center of the attachment of the LCL and MCL during surgical repair of these ligaments.

Espregueira-Mendes and da Silva (2006) examined the histology of the LCL from 20 male cadavers with the mean age of 50 years. They reported that the length of the anterior bundle of the LCL was $63 \mathrm{~mm}$. In the present study we used 6 male and 17 female cadavers with the mean age of 79.5 years. The length of the anterior bundle of the LCL was shorter than that of their report. The length depends on the sex and age of the cadavers. The length of the anterior and posterior bundle of the MCL was reported to be about $85 \mathrm{~mm}$ and $100 \mathrm{~mm}$ respectively (Nomura et al., 1989; Manabe et al., 1990). Similar results were found in the present study. From the above results, both anterior and posterior bundles of the MCL were longer than those of the LCL. The posterior bundle length was longer than the anterior one. This difference causes the movement variation of the anterior and posterior bundles at knee flexion (Bartel et al., 1977). It was reported that the anterior bundle of the LCL became longer and the posterior one became shorter with the knee flexion (Wang et al., 1974; Park et al., 2005). The length of the anterior bundle of the MCL had no marked change and the posterior bundle became consistently shorter with the knee flexion, suggesting that the posterior bundle of the MCL might be tight in the full extension (Park et al., 2005). Ries et al. (2003) stated that the medial and lateral soft tissue balance was dependent on the length of the collateral ligaments. The soft tissue constraint in flexion was dependent on the structural integrity of the collateral ligaments. When one of the collateral ligaments loosened, the soft tissue balance might not be achieved during the knee flexion.

As to the size of the proximal attachments, the longitudinal diameter of the LCL was longer than the transverse one (Espregueira-Mendes and da Silva, 2006). However, the longitudinal diameter of the MCL was shorter than the transverse one (Suda et al., 1992). For the distal attachment, the longitudinal diameter of the LCL and MCL was longer than that of the transverse one (Suda et al., 1992; Espregueira-Mendes and da Silva, 2006). The size of the distal attachment of the MCL was largest, and the size of the proximal attachment of the LCL was larger than that of the distal one. Ligamentous reconstruction might be performed by using autograft or allograft tendons for collateral ligamentous instability and the autograft or allograft should be fixed at the normal attachment sites to restore isometry (Nelson et al., 2003). Our results may be beneficial in deciding the fixation point and size of the ligaments.

The proximal and the distal bundles of the LCL showed forward inclination same as the Suda's report (1992). The distal bundle of the MCL demonstrated the backward inclination. It was reported that the inclination of the LCL changed from forward to backward with the knee flexion, while the MCL showed backward inclination even though the knee was flexed (Kapandji, 1983; Meister et al., 2000).

As for the LCL, the inclination of the proximal attachment bundle and the inclination of the distal attachment bundle and the inclination of the longitudinal axis of the LCL was almost same. As for the MCL, the inclination of the distal attachment bun- 
dle was similar to the inclination of the longitudinal axis of the MCL. But the inclination of the proximal attachment bundle was different from the inclination of the distal attachment bundle and the inclination of the longitudinal axis of the MCL. Future examination is needed about this point.

In conclusion, the present study demonstrated that the MCL was longer than the LCL. The proximal attachment of the MCL was more anterior and superior than that of the LCL. As compared with the LCL, the distal attachment of the MCL located a little forward and inferior. The inclination of the MCL was backward, while that of the LCL was forward. The inclination of the proximal and distal attachment bundles of the LCL was similar to the inclination of the longitudinal axis of the LCL. The inclination of the proximal attachment bundle was different from both the inclination of the distal attachment bundle and the inclination of the longitudinal axis of the MCL. These results showed that the morphological properties of the LCL and MCL were different. It may provide a better understanding of the function of the collateral ligaments.

\section{References}

1) Bartel DL, Marshall JL, Schieck RA and Wang JB. Surgical repositioning of the medial collateral ligament. J Bone Joint Surg Am 1977; 59:107-116.

2) Espregueira-Mendes and da Silva MV. Anatomy of the lateral collateral ligament: a cadaver and histological study. Knee Surg Sports Traumatol Arthrosc 2006; 14:221-228.

3) Fetto JF and Marshall JL. Medical collateral ligament injuries of the knee: A Rationale for Treatment. Clin Orthop Relat Res 1978; 132:206-218.

4) Grood ES, Stowers SF and Noyes FR. Limits of movement in the human knee. J Bone and Joint Surg Am 1988; 70-A:88-97.

5) Kapandji IA. The knee. In: Kapandji IA, ed, The physiology of the joints, 2nd ed. London, Churchill Livingstone, $1983 ; 72-135$.

6) Kennedy JC, Hawkins RJ, Willis RB and Danylchuk KD. Tension Studies of Human Knee Ligaments. J Bone Joint Surg Am 1976; 58:350-355.

7) Liow RYL, McNicholas MJ, Keating JF and Nutton RW. Ligament repair and reconstruction in traumatic dislocation of the knee. J Bone Joint Surg Br 2003; 85-B:845-851.

8) Manabe H, Ikeda A, Ochi M and Ikuta Y. Anatomical study of medial collateral ligament of the knee (in Japanese). J Jpn Orthop Assoc 1990; 64:1193.

9) Meister BR, Michael SP, Moyer RA, Kelly JD and Schneck $\mathrm{CD}$. Anatomy and kinematics of the lateral collateral ligament of the knee. Am J Sports Med. 2000; 28:869-878.
10) Murase K, Kumano K, Bannouji T, Yokoe K, Kaneko K, Irie K, Ookubo F and Nakajima H. Injury of medial collateral ligament of the knee (in Japanese). Orthop Surg Traumatol 1985; 28:23-29.

11) Nelson CL, Gioe TJ, Cheng EY and Thompson RC. Implant selection in revision total knee arthropasty. J Bone Joint Surg Am 2003; 85-A:43-51.

12) Nomura E, Fujikawa $K$, Isekame $F$, Takeda $T$, Matsumoto $\mathrm{H}$ and Ootani T. Anatomical study of medial collateral ligament of the knee (in Japanese). J Jpn Orthop Assoc 1989; 63:1086.

13) Pasque C, Noyes FR, Gibbons M, Levy M and Grood E. The role of the popliteofibular ligament and the tendon of popliteus in providing stability in the human knee. J Bone Joint Surg Br 2003; 85-B:292-298.

14) Park SE, DeFrate LE, Suggs JF, Gill TJ, Rubash HE and $\mathrm{Li} \mathrm{G}$. The change in length of the medial and lateral collateral ligaments during in vivo knee flexion. The Knee 2005; 12:377-382.

15) Ries MD, Haas SB and Windsor RE. Soft-tissue balance in revision total knee arthroplasty. J Bone Joint Surg Am 2003; 85-A:38-42.

16) Robinson JR, Sanchez-Ballester J, Bull AMJ, Thomas R de $\mathrm{WM}$ and Amis AA. The posteromedial corner revisited. An anatomical description of the passive restraining structures of the medial aspect of the human knee. J Bone Joint Surg Br 2004; 86-B:674-681.

17) Robinson JR, Bull AMJ and Amis AA. Structural properties of the medial collateral ligament complex of the human knee. J Biomech 2005; 38:1067-1074.

18) Shahane SA, Ibbotson $C$, Strachan $R$ and Bickerstaff DR. The popliteofibular ligament. J Bone Joint Surg Br 1999; 81-B:636-642.

19) Suda Y, Fujikawa K, Matsumoto H, Takeda T and Miyasaka T. Anatomical study on the lateral structures of the knee joint - with special reference to the lateral collateral ligament (in Japanese). Cent Jpn J Orthop Traumat 1992; 35:171-172.

20) Suda Y, Fujikawa K, Matsumoto H, Takeda T and Kurimura M. Anatomical study on the lateral structures of the knee joint - with special reference to length pattern of the lateral collateral ligament (in Japanese). Cent Jpn J Orthop Traumat 1993; 36:1413-1414.

21) Sugita $T$ and Amis AA. Anatomic and biomechanical study of the lateral collateral and popliteofibular ligaments. Am J Sports Med. 2001; 29:466-472.

22) Wang CJ and Walker PS. Rotatory laxity of the human knee joint. J Bone Joint Surg Am 1974; 56-A:161-170.

23) Warren LF, Marshall JL and Girgis F. The Prime Static Stabilizer of the Medial Side of the Knee. J Bone Joint Surg Am 1974; 56-A:665-674.

24) Warren LF and Marshall JL. The Supporting Structures and Layers on the Medial Side of the knee. J Bone Joint Surg Am 1979; 61-A:56-62.

25) Wymenga AB, Kats JJ, Kooloos J and Hillen B. Surgical anatomy of the medial collateral ligament and the posteromedial capsule of the knee. Knee Surg Sports Traumatol Arthrosc 2006; 14:229-234. 